\title{
Thermal Expansion Contribution to the Temperature Dependence of Excitonic Transitions in GaAs and AlGaAs
}

\author{
S. A. Lourenço, ${ }^{1}$ I. F. L. Dias,${ }^{1}$ J. L. Duarte, ${ }^{1}$ E. Laureto, ${ }^{1}$ \\ L. C. Poças, ${ }^{1}$ D. O. Toginho Filho, ${ }^{1}$ and J. R. Leite, ${ }^{2}$ \\ ${ }^{1}$ Departamento de Física, Universidade Estadual de Londrina, UEL, CP6001, CEP 86051-970, Londrina, PR, Brazil \\ ${ }^{2}$ Instituto de Física, Universidade de São Paulo, IFUSP, CP 66318, São Paulo, 05315-970, SP, Brazil
}

Received on 21 July, 2003. Revised version received on 5 November, 2003

\begin{abstract}
Photoluminescence and photoreflectance measurements have been used to determine excitonic transitions in the ternary $\mathrm{Al}_{x} \mathrm{Ga}_{1-x} \mathrm{As}$ alloy in the temperature range from 2 to $300 \mathrm{~K}$. The effect of the thermal expansion contribution on the temperature dependence of excitonic transitions for different aluminum concentrations in the $\mathrm{Al}_{x} \mathrm{Ga}_{1-x}$ As alloy is presented. Results from this study have shown that the negative thermal expansion (NTE) in the $\mathrm{Al}_{x} \mathrm{Ga}_{1-x} \mathrm{As}$ alloy, in the low temperature interval, induces a small blueshift in the optical transition energy. In the temperature range from $\sim 23$ to $\sim 95 \mathrm{~K}$ there is a competition between the NTE effect and the electron-phonon interaction. Using the thermal expansion coefficient in the $2-300 \mathrm{~K}$ temperature range, the thermal expansion contribution to GaAs, at room temperature, represents $21 \%$ of the total shift of the excitonic transition energy. After subtracting the thermal expansion contribution from the experimental temperature dependence of the excitonic transitions, in the $\mathrm{Al}_{x} \mathrm{Ga}_{1-x} \mathrm{As}$ alloy, the contribution to the electron-phonon interaction of the longitudinal optical phonon increases, relatively to the longitudinal acoustical phonon, with increasing $A l$ concentration.
\end{abstract}

\section{Introduction}

The GaAs and its related alloy $\mathrm{Al}_{x} \mathrm{Ga}_{1-x} \mathrm{As}$ form a very important family of semiconductor materials used as active elements in many types of high performance optoelectronic and high-speed electronic devices [1,2]. One of the most important parameter of any semiconductor material is the band-gap energy. In $\mathrm{Al}_{x} \mathrm{Ga}_{1-x}$ As alloys, the lowest bandgap energy at low aluminum concentration, $0 \leq x \leq 0.45$, is a direct energy gap located at the $\Gamma$ point of the Brillouin zone, and its temperature and aluminum dependency have been the focus of many researches in the last years. The temperature dependence of the band-gap energy, in special, can be explained by the sum of two distinct mechanisms: the electron-phonon interaction and the lattice thermal expansion. The main contribution to the temperature dependence of the band-gap energy is attributed to electron-phonon interactions [3-6]. This effect can also be broken up into two contributions: the effect of the second-order electronphonon interaction taken to first order in perturbation theory - the so-called Debye-Waller terms, and the effect of the first-order electron-phonon interaction taken to second order in perturbation theory - the Fan terms [4,5]. According to some researches, the contribution of the thermal expansion at room temperature varies between $2 \%$ and $20 \%$ in most of the semiconductor materials [6,7]. However, in the GaAs and $\operatorname{In}_{x} \mathrm{Ga}_{1-x}$ As with low indium concentration $(x=$ 0.06 and $x=0.15$ ), the thermal expansion mechanism contributes with $40 \%$ to $45 \%$ to the total band-gap energy reduction $[8,9]$, which is higher than the contribution of other semiconductor materials. Despite this significant contribution to the temperature dependence in the band-gap energy or its related excitonic transition energy, the thermal expansion mechanism is not, generally, taken into account in the analysis of the temperature dependence of the band-gap energy [9-13].

Furthermore, an interesting phenomenon related to the lattice thermal expansion, the negative thermal expansion (NTE), takes place in many tetrahedral semiconductors at low temperatures [14-17]. This apparent anomalous behavior is observed in many direct band-gap semiconductors as the binary materials of III-V group, GaAs [15], InP [15], $\mathrm{AlSb}$ [21], GaSb [21], InAs [22], and InSb [23]; and in the binary and ternary materials of II-VI group, $\mathrm{Hg}_{1-x} \mathrm{Cd}_{x} \mathrm{Te}$ and $\mathrm{Hg}_{1-x} \mathrm{Zn}_{x} \mathrm{Te}$ [24], as well as in semiconductor materials with indirect band-gap energy as $\mathrm{Si}$ [18]. This nonmonotonic dependence on $\mathrm{T}$ is attributed to the negative Grüneissen parameters of the transverse acoustical (TA) phonon branches near the limit of the Brillouin-zone [14$16,19,20]$. As well as the thermal expansion mechanism, the effect of the negative thermal expansion on semiconductor materials has not been generally take into account in the analysis of the temperature dependence of the band-gap energy.

In this work, we present a study of the optical transitions temperature dependence in the GaAs and $\mathrm{AlGaAs}$ in the temperature range from 2 to $300 \mathrm{~K}$. Photoluminescence (PL) and photoreflectance (PR) techniques are used to study the optical transitions in bulk GaAs and $\mathrm{Al}_{x} \mathrm{Ga}_{1-x}$ As at aluminum concentration varying between 0.17 and 0.40 . The 
Viña [25] and Pässler [9,26] models are used to fit the temperature dependence of the excitonic transitions energy. The effect of the thermal expansion (TE) on all the temperature range $(2-300 \mathrm{~K})$ and of the NTE on the low temperature range $(\mathrm{T}<100 \mathrm{~K})$ are analyzed. We show that it is necessary to take into account the thermal expansion coefficient in all the temperature range $(0-300 \mathrm{~K})$ for the present analysis of the temperature dependence of the band-gap energy for AlGaAs semiconductor materials in the $2-300 \mathrm{~K}$ temperature range.

\section{Experimental details}

Three nominally undoped $0.3-\mu$ m-thick AlGaAs samples were grown by molecular-beam epitaxy (MBE) on a $0.2-$ $\mu$ m-thick GaAs buffer and on an undoped semi-insulating (100)-GaAs substrate. The aluminum compositions were $0.17,0.30$ and 0.40 . The experimental data at $x=0$ were redigitalized from the work of Lautenschager et al. [27]. Excitonic transitions in AlGaAs alloy were determined by $\mathrm{PL}$ at temperatures up to $130 \mathrm{~K}$ and by PR at higher temperatures. The photoluminescence measurements were carried out between 2 and $130 \mathrm{~K}$ in a He flow cryostat (JANIS, Optical SuperVari.Temp. model 7.7C) equipped with a Lake Shore temperature controller (Model 805) using the $441.6 \mathrm{~nm}$ line of an He-Cd laser as the excitation source, with an intensity of $\sim 20 \mathrm{~W} / \mathrm{cm}^{2}$. The emission spectra were analyzed with a $0.5 \mathrm{~m}$ monochromator and detected by a liquid N2 cooled Ge photodetector, using standard lock-in techniques. The photoreflectance was measured with a conventional system consisting of a white light from a tungsten lamp disperse through a $0.5 \mathrm{~m}$ focal length monochromator shining onto the sample at an incident angle of approximately $45^{\circ}$. The optical modulation was provided by the $632.8 \mathrm{~nm}$ line of an He-Ne laser beam mechanically chopped at a frequency $f=200 \mathrm{~Hz}$ and incident at an angle of $30^{\circ}$. A long pass filter of $665 \mathrm{~nm}$ cut off was used to prevent scattered radiation from the laser to overwhelm the detector. Specular reflection light was detected using a $\mathrm{Si}$ photodiode, whose signal was fed in a lock-in amplifier (STANFORD SR 510) tuned to $200 \mathrm{~Hz}$. The signal was processed by a microcomputer to obtain $\Delta R / R$ as a function of photon energy. Temperature dependent PR measurements were carried out by mounting the sample on the cold finger of a liquid nitrogen variable temperature cryostat, whose temperature can be varied between 85 and $330 \mathrm{~K}$. The sample temperature was measured from $130 \mathrm{~K}$ to $300 \mathrm{~K}$ with a calibrated copper-constantan thermocouple in good thermal contact with the sample. The uncertainty in the temperature determination is estimated to be $\pm 1.5 \mathrm{~K}$ and the temperature stability during each spectrum was better than $\pm 1 \mathrm{~K}$. The composition of the layer was determined by the Double Crystal X-Ray Diffraction at a $0.5 \%$ accuracy rate [28].

\section{Theoretical models}

Many analytical models used to describe the temperature dependence of the band-gap energy have been developed in the last decades [9,25]. A recent work [29] pointed out that the Viña et al.[25] and the Pässler [9] models are the most suitable ones to fit the temperature dependence either of the band-gap energy or of the excitonic transition energy. The Viña approach is defined by the expression:

$$
E_{g}(T)=E_{B}-a_{B}\left[1+\frac{2}{\exp \left(\Theta_{B} / T\right)-1}\right]
$$

where $a_{B}$ represents the strength of the electron-phonon interaction, $\Theta_{B} \equiv \hbar w / k_{B}$ is the characteristic temperature parameter representing the effective phonon energy on the temperature scale $[11,25]$ and $E_{g}(T=0)=E_{B}-a_{B}$. The Pässler [9] model is characterized by the expression:

$$
E_{g}(T)=E_{g}(T=0)-\frac{\alpha \Theta}{2}\left[\sqrt[p]{1+\left(\frac{2 T}{\Theta}\right)^{p}}-1\right]
$$

where $E_{g}(T=0)$ is the energy gap at zero Kelvin temperature, $\alpha \equiv S(\infty) \equiv-(d E(T) / d T)_{T \longrightarrow \infty}$ is the high-temperature limit value for the forbidden gap entropy [30,31], $\Theta$ is a characteristic temperature parameter of the material representing the effective phonon energy $\hbar w \equiv$ $k_{B} \Theta$ in units of absolute temperature (similar to the $\Theta_{B}$ defined for the Viña model), and $p$ is an empirical parameter related to the shape of the electron-phonon spectral functions $[9,32]$. In this work, $E_{g}(T)$ represents the excitonic transitions energy in bulk GaAs and AlGaAs. We work with excitons in the GaAs and AlGaAs alloys to obtain direct experimental evidence of transitions and to avoid possible errors due to the composition dependence of the excitonic binding energies in the these alloys [10]. The least-square fit was used as an accuracy criterion in the description of the experimental data point fits.

The variation of the band-gap energy with temperature induced by thermal expansion, $\Delta E_{t h}(T)$, can be written as [8,33-35]:

$$
\Delta E_{t h}(T)=-3 B\left(\frac{\partial E}{\partial P}\right)_{T} \int_{0}^{T} \alpha_{t h}\left(T^{\prime}\right) d T
$$

where $\mathrm{B}$ is the bulk modulus, $d E / d p$ is the pressure-induced band-gap shift and $\alpha_{t h}(T)$ is the linear thermal expansion coefficient. Taking into account the contribution of the TE, the equations (1) and (2) can be rewritten as:

$$
E_{g}(T)-\Delta E_{t h}(T)=E_{B}^{\prime}-a_{B}^{\prime}\left[1+\frac{2}{\exp \left(\Theta_{B}^{\prime} / T\right)-1}\right]
$$

and

$$
E_{g}(T)-\Delta E_{t h}(T)=E_{g}^{\prime}(T=0)-\frac{\alpha^{\prime} \Theta}{2}\left[\sqrt[p]{1+\left(\frac{2 T}{\Theta}\right)^{p}}-1\right]
$$


The parameters $E_{B}^{\prime}, a_{B}^{\prime}, \Theta_{B}^{\prime} ; E_{g}^{\prime}(T=0), \alpha^{\prime}, \Theta$, obtained from the fit of these expressions are related exclusively to the electron-phonon interaction mechanism of the semiconductor material.

\section{Results and discussion}

Figure 1(A) shows the PL spectra for the $\mathrm{Al}_{x} \mathrm{Ga}_{1-x}$ As alloy with $x=0.30$ at $2 \mathrm{~K}, 77 \mathrm{~K}$, and $130 \mathrm{~K}$. The PL peak energy at $1.889 \mathrm{eV}$, with $7.1 \mathrm{meV}$ of full width at half maximum (FWHM), is identified as being due to the bound exciton (BE) recombination. At low temperatures, the PL spectrum of the alloy is dominated by the excitonic recombination. At $77 \mathrm{~K}$, the thermal population of the higher energy states is favored, thus, the band-to-band recombination is observed. Both excitonic and band-to-band recombination energy peak position were obtained by the least-square fits, using a number of appropriate transitions. These transitions are represented by the dotted lines in Fig. 1(A). The solid lines represent the total fits (linear superposition of the dotted lines). Even with the strong contribution of the band-to-band recombination at higher temperatures, the excitonic recombination can still be identified. These results are similar to those obtained by Grilli et al.[12] for the GaAs. The PL peak energy at $1.869 \mathrm{eV}$ with $28.2 \mathrm{meV}$ of FWHM, is associated with the recombination due to impurity states. The recombination involving the impurities has its intensity reduced by the increase in the temperature, disappearing at temperatures above $120 \mathrm{~K}$. This is a classical behavior of impurity and has been observed by many research groups [36-38]. Additionally, the impurity nature of the lower energy PL peak was verified by analyzing its behavior as a function of the excitation intensity (not shown in this paper). It is known that in MBE grown films carbon impurities incorporate into the lattice and produce an acceptor state approximately $20 \mathrm{meV}$ below the GaAs gap [3638]. In our samples, the acceptor impurities are identified by its position in energy as being carbon $\left(\mathrm{e}, \mathrm{A}^{\mathrm{o}}\right)$. The signal obtained by PL technique decreases in temperatures above $150 \mathrm{~K}$, making difficult to identify the transitions properly. So, the PR technique was used in the 130 to $300 \mathrm{~K}$ temperature range. The Fig. 1(B) shows the PR experimental spectra at different temperatures. Experimental line shape in low field PR can be fitted by expression of the type [3941]: $\Delta R / R=\operatorname{Re}\left[C e^{i \varphi}\left(E-E_{g}+i \Gamma\right)^{-n}\right]$, where $C$ is an amplitude coefficient, $\varphi$ is a phase factor, $E$ is the photon energy, $E_{g}$ is the transition energy and $\Gamma$ is the broadening parameter. The exponent $n$ is a parameter that depends on the type of critical point. According to Aspnes [39], $n=$ 2, 2.5 and 3 for an exciton or impurity in bulk material, a $3 \mathrm{D}$ band-to-band, and a 2D band-to-band transition, respectively. The least-square fits of the PR spectrum presents two components that are quite close in energy at all measured temperatures. These components have been observed in all AlGaAs samples discussed in this text. The inset of Fig. $1 \mathrm{~B}$ shows these components for sample \#3 at $300 \mathrm{~K}$. These components were identified as conduction-band-to-acceptor and excitonic transitions. Several research groups have also reported the observation of impurity and excitonic components in the PR spectra at room temperature [42-47].

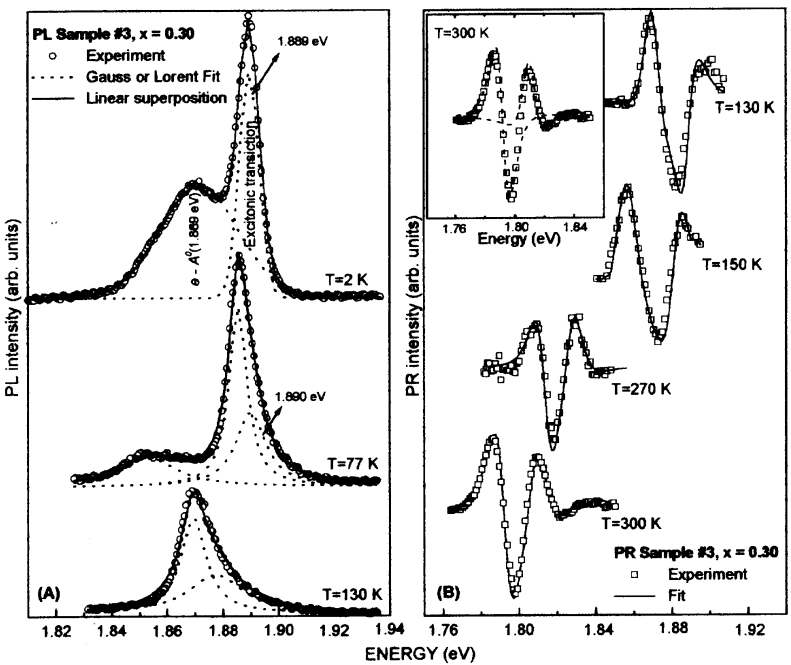

Figure 1. PL and PR spectra of the $\mathrm{Al}_{0.30} \mathrm{Ga}_{0.70} \mathrm{As}$ alloy are shown at different temperatures. Fig.1(A) shows the PL spectra at 2, 77 and $130 \mathrm{~K}$. At $2 \mathrm{~K}$ there are two radiative channels (dotted lines) identified as the recombination from the conduction band to the carbon acceptor state with peak at $1.869 \mathrm{eV}$ and the bound exciton recombination with peak at $1.889 \mathrm{eV}$. At $77 \mathrm{~K}$ another radiative channel appears along the side of highest energy, identified as the band-to-band transition. The solid lines are the total fit for the experimental data (linear superposition of the dotted lines). Fig.1(B) shows the PR spectra for the following temperatures:130, 150, 270 and $300 \mathrm{~K}$. The squares are the experimental points and the solid lines are the least-square fits using the line shape $\Delta R / R=\operatorname{Re}\left[C e^{i \varphi}\left(E-E_{g}+i \Gamma\right)^{-n}\right][39-41]$.

Due to the different physical processes involved in the different techniques, a difference in energy, called "Stokes shift", is expected. However, some researchers have observed that, either at sufficiently high-excitation intensity or at adequately high temperatures [48-51], the energy difference between the photoluminescence spectrum and the absorption (or photoreflectance) spectrum of some semiconductor alloys with a random distribution, such as the $\mathrm{Al}_{x} \mathrm{Ga}_{1-x} \mathrm{As}$, is nearly null. In this work the photoreflectance spectra were obtained for temperatures over 130 $\mathrm{K}$ and for alloy with a random distribution (homogeneous samples) [50]. Therefore, a negligible Stokes shift between the PL and PR spectra has been observed [29].

Figure 2 shows the temperature dependence of excitonic transitions in the $\mathrm{GaAs}$ and $\mathrm{Al}_{x} \mathrm{Ga}_{1-x}$ As alloys at $x=0.17$, $0.30,0.40$ in the temperature range from 2 to $300 \mathrm{~K}$, and the numerical fit based on the Pässler model. Values for the Viña and Pässler parameters are shown in Table I. Both models give similar fittings to the experimental points of the excitonic transition dependence with temperature. With increasing aluminum concentration in the AlGaAs ternary alloy, an increase in the adjustment parameters of the Viña and Pässler models can be observed. This behavior of the adjustment parameters, due to the aluminum concentration in AlGaAs, was discussed in a recent article [29]. 
TABLE I. Parameters of the Viña and Pässler expressions obtained from temperature dependence fits of excitonic transitions in the $\mathrm{Al}_{x} \mathrm{Ga}_{1-x}$ As and GaAs materials.

\begin{tabular}{|c|c|c|c|c|c|}
\hline \multirow{2}{*}{ Sample } & $\begin{array}{c}E_{B}(\mathrm{meV}) \\
E_{g}(T=0)(\mathrm{meV})\end{array}$ & $\begin{array}{c}a_{B}(\mathrm{meV}) \\
\alpha\left(10^{-4} \mathrm{eV} / \mathrm{K}\right)\end{array}$ & $\begin{array}{c}\Theta_{B}(\mathrm{~K}) \\
\Theta(\mathrm{K})\end{array}$ & $\begin{array}{c}k_{B} \Theta_{B}(\mathrm{meV}) \\
k_{B} \Theta(\mathrm{meV})\end{array}$ & $p$ \\
\hline \multirow{2}{*}{ \#1 $(x=0)$} & $1576.6 \pm 1.6$ & $59.7 \pm 7.4$ & $241 \pm 7$ & 20.7 & - \\
& $1517.6 \pm 1.1$ & $4.9 \pm 0.2$ & $203 \pm 8$ & 17.5 & 2.85 \\
\hline \multirow{2}{*}{$\# 2(x=0.17)$} & $1802.4 \pm 4.1$ & $76.9 \pm 4.4$ & $275 \pm 11$ & 23.7 & - \\
& $1725.1 \pm 0.6$ & $5.1 \pm 0.1$ & $208 \pm 9$ & 17.9 & 4.0 \\
\hline \multirow{2}{*}{$\# 3(x=0.30)$} & $1975.7 \pm 2.2$ & $85.9 \pm 2.3$ & $289 \pm 6$ & 24.9 & - \\
& $1890.0 \pm 0.3$ & $5.5 \pm 0.1$ & $220 \pm 7$ & 18.9 & 3.5 \\
\hline \multirow{2}{*}{$\# 4(x=0.40)$} & $2081.2 \pm 4.6$ & $94.4 \pm 4.8$ & $317 \pm 10$ & 27.3 & - \\
& $1989.7 \pm 0.6$ & $5.9 \pm 0.1$ & $275 \pm 9$ & 23.7 & 2.9 \\
\hline
\end{tabular}

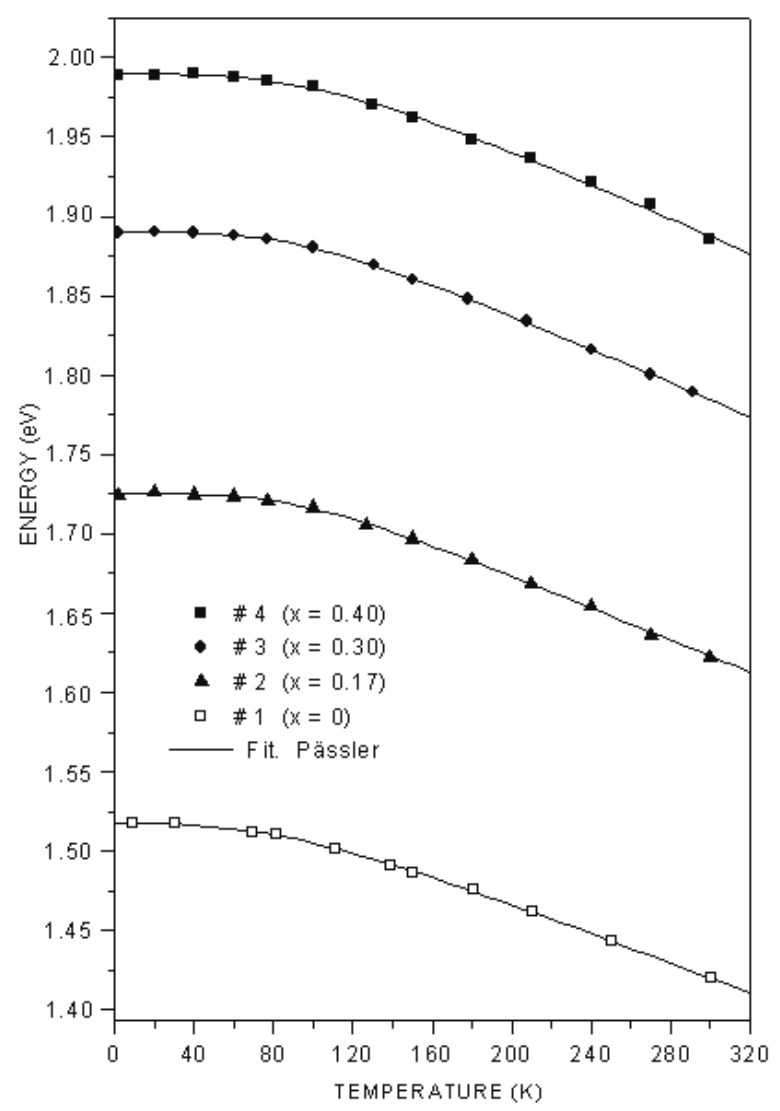

Figure 2. The experimental temperature dependence of the excitonic transitions in $\mathrm{Al}_{x} \mathrm{Ga}_{1-x}$ As samples (\#1, \#2, \#3, \#4) at different aluminum compositions $(x=0.0,0.17,0.30,0.40)$. The solid lines are least-squares fits of the Pässler model [Eq.(2)].

A large series of experimental papers have discussed the fitting of the temperature dependence of excitonic transitions in semiconductor materials within the framework of the electron-phonon interaction only (the thermal expansion mechanism of the lattice is not taken into account) [9,10,11,32]. Moreover, the negative thermal expansion phenomenon can give important contributions to the variation of the excitonic transitions energy with temperature in the low temperatures range $(\mathrm{T}<100 \mathrm{~K})$. The importance of this contribution to GaAs is shown in Fig. 3. Fig. 3A shows the experimental data points of the thermal expan- sion coefficients, $\alpha_{t h}(T)$, for the GaAs, obtained by Soma et al.[15]. A good fit for the thermal expansion coefficient was obtained using a polynomial expansion under the following condition: $\alpha_{t h}=0$ and $d \alpha_{t h} / d T=0$ at $T=0$ $\mathrm{K}$. The negative thermal expansion is observed in the temperature range from 10 to $70 \mathrm{~K}$, reaching its maximum at $T \cong 45 K\left(\alpha_{t h} \cong-0.5 \cdot 10^{-6} K^{-1}\right)$. The energy shift, $\Delta E_{t h}(T)$, associated with the thermal expansion is shown in Fig. 3B. The solid curve in Fig. 3B represents the calculation of the energy shift, using expression (3) with the thermal expansion coefficient, $\alpha_{t h}(T)$, as a function of temperature as shown in Fig. 3A. The energy variation induced by the TE, considering $\alpha_{t h}(T)$, is practically null up to $\sim 23$ $\mathrm{K}$. In the temperature range from 23 to $61 \mathrm{~K}$, however, it increases due to the NTE effect, and finally decreases at higher temperatures. The dash-dotted line represents $\Delta E_{t h}(T)$ calculated by the expression (3) with $\alpha_{t h}(T=300 K)$. The energy variation with temperature induced by the TE, considering $\alpha_{t h}(T=300 K)$, is linear and decreases in all the temperatures range. The approach that considers a constant value $\alpha_{t h}(T=300 K)$ at all temperature interval has been discussed in several papers $[8,52,53]$. A large difference between the two curves can be seen in almost all the temperature range, except at the $T=0 K$ point. The difference between the two curves increases up to $\sim 135 \mathrm{~K}$ and tends to display a constant value of approximately $18.5 \mathrm{meV}$ at higher temperatures.

The temperature dependence of the excitonic transitions for GaAs in the $2-300 \mathrm{~K}$ temperature range is shown in Fig. 4. The solid squares represent the experimental data of the excitonic transitions dependence with temperature. The circles [triangles] represent the experimental points obtained by subtracting $\Delta E_{t h}(T)$ using $\alpha_{t h}(T)\left[\alpha_{t h}(T=300 K)\right]$ from the experimental values of $E_{g}(T)$. The solid line represents the fit of the Pässler expression for the experimental points of the excitonic transition energy dependence with temperature and the dash-dotted line represents the fit of the Pässler approach after the subtraction of the thermal expansion contribution. The results of the Viña and Pässler parameters obtained by fitting the experimental points after the subtraction of the thermal expansion contribution are showed in Table II. To obtain optimized fittings for the variation of the excitonic transitions with temperature after subtracting the thermal expansion contribution, the values of the 
TABLE II. Parameters of the Viña and Pässler models obtained from temperature dependence fits of excitonic transitions in the $\mathrm{Al}_{x} \mathrm{Ga}_{1-x} \mathrm{As}$ at $x=0.0,0.17,0.30$, and 0.40 compositions, taking into account the thermal expansion contribution effects.

\begin{tabular}{|c|c|c|c|c|c|}
\hline \multirow{2}{*}{ Sample } & $\begin{array}{c}E_{B}^{\prime}(\mathrm{meV}) \\
E_{g}^{\prime}(T=0)(\mathrm{meV})\end{array}$ & $\begin{array}{c}a_{B}^{\prime}(\mathrm{meV}) \\
\alpha^{\prime}\left(10^{-4} \mathrm{eV} / \mathrm{K}\right)\end{array}$ & $\begin{array}{c}\Theta_{B}^{\prime}(\mathrm{K}) \\
\Theta^{\prime}(\mathrm{K})\end{array}$ & $\begin{array}{c}k_{B} \Theta_{B}^{\prime}(\mathrm{meV}) \\
k_{B} \Theta^{\prime}(\mathrm{meV})\end{array}$ & $p$ \\
\hline \multirow{2}{*}{$\# 1(x=0)$} & $1547.5 \pm 4.5$ & $30.2 \pm 0.5$ & $175 \pm 2$ & 15.1 & - \\
& $1517.6 \pm 0.06$ & $3.4 \pm 0.01$ & $153 \pm 1$ & 13.2 & 2.85 \\
\hline \multirow{2}{*}{ \#2 $(x=0.17)$} & $1776.2 \pm 1.7$ & $51.1 \pm 1.9$ & $251 \pm 6$ & 21.6 & - \\
& $1725.0 \pm 0.09$ & $3.7 \pm 0.01$ & $183 \pm 14$ & 15.7 & 4.0 \\
\hline \multirow{2}{*}{$\# 3(x=0.30)$} & $1944.7 \pm 1.1$ & $54.9 \pm 1.1$ & $253 \pm 4$ & 21.8 & - \\
& $1889.9 \pm 0.3$ & $4.0 \pm 0.09$ & $194 \pm 1$ & 16.7 & 3.5 \\
\hline \multirow{2}{*}{$\# 4(x=0.40)$} & $2048.0 \pm 2.3$ & $59.0 \pm 2.5$ & $276 \pm 7$ & 23.8 & - \\
& $1989.5 \pm 0.09$ & $4.2 \pm 0.05$ & $240 \pm 2$ & 20.7 & 2.9 \\
\hline
\end{tabular}
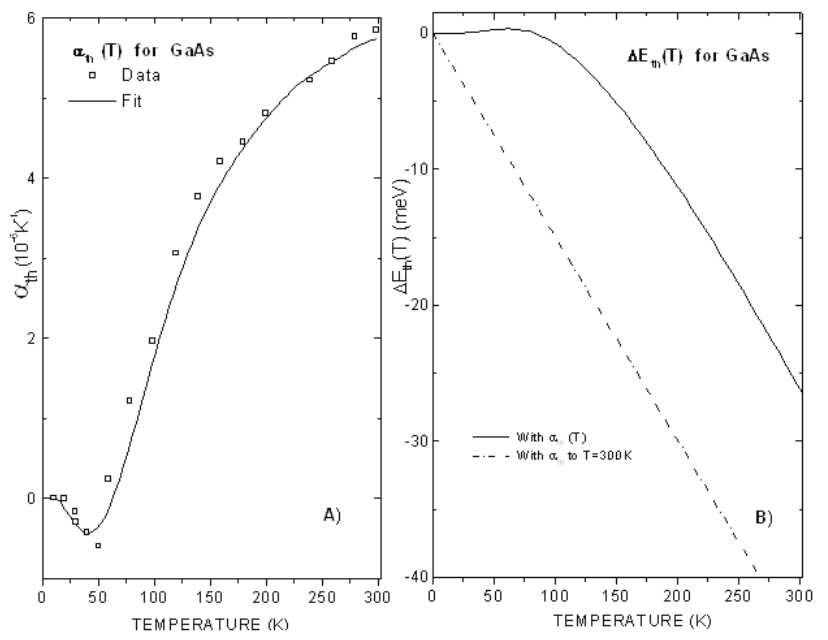

Figure 3. Fig. 3A shows the temperature dependence of the linear thermal expansion coefficient for the GaAs obtained by Soma et al. (open square) [15]. The solid curve represents a fit of experimental points using a polynomial expansion. Fig. 3B shows the energy shift $\Delta E_{t h}(T)$ defined by the equation (3) associated to the temperature dependence of the thermal expansion. The solid and dash-dotted curves represent the curves using $\Delta E_{t h}(T)$, estimated with a temperature dependence thermal expansion coefficient $\alpha_{t h}(T)$ and with a constant thermal expansion coefficient $\alpha_{t h}(T=300 K)$, respectively.

parameter $p$ were kept the values obtained before subtracting the thermal expansion contribution. The fitting of the $\left(E_{g}-\Delta E_{t h}\right)$ curve by the Pässler and the Vinã expressions, using $\alpha_{t h}(T=300 K)$, is not as good as that using $\alpha_{t h}(T)$. The $E_{g}(T)$ values, minus the energy shift $\Delta E_{t h}(T)$, calculated by the different approaches $\left[\alpha_{t h}(T=300 K)\right.$ or $\alpha_{t h}(T)$ ], differ considerably in all the temperature range. Different values of the thermal expansion contribution to the shift of the band-gap energy in GaAs at $T=300 \mathrm{~K}$ has been reported in the literature. Walter et al. [7], for example, obtained a contribution of only 13\%. However, as observed by Gopalan et al. [54], Walter's calculations completely ignored the contribution of the Fan term to the shift of the band gap with $T$. This result overestimates the shift of the band gap in GaAs by about $60 \mathrm{meV}$ when the temperature is

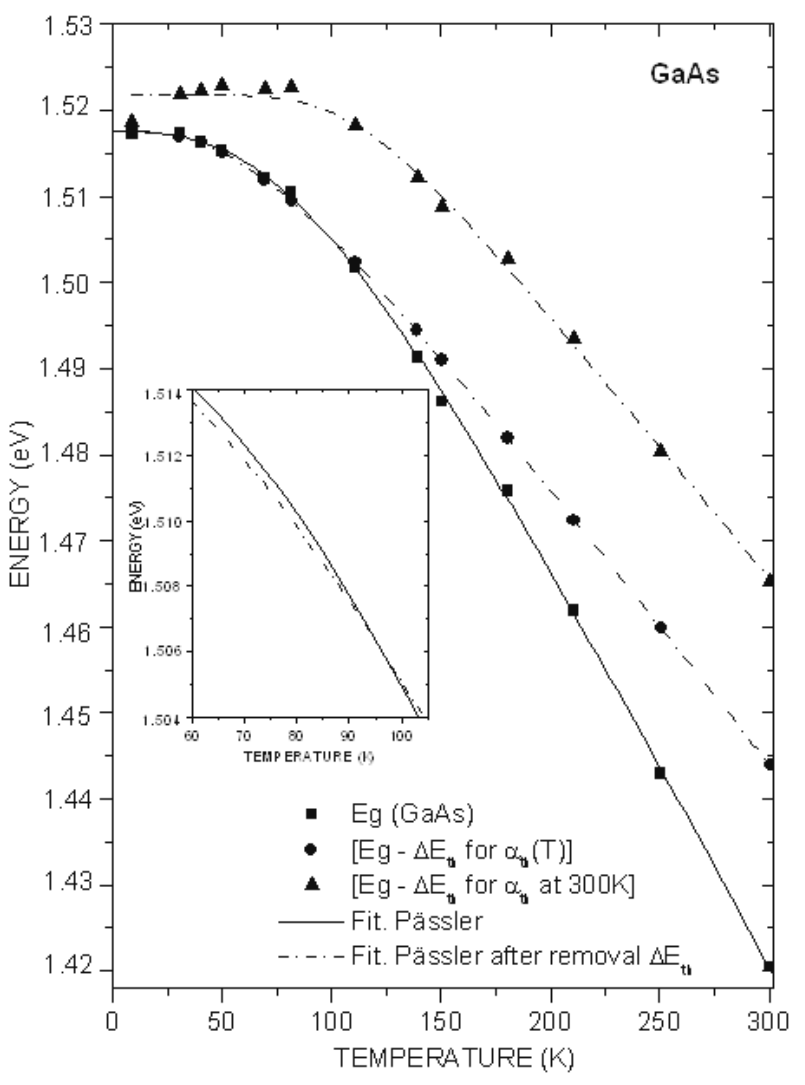

Figure 4. Temperature dependence of the GaAs excitonic transitions (solid square). The circles and triangles represent the experimental points minus $\Delta E_{t h}(T)$ using $\alpha_{t h}(T)$ and $\alpha_{t h}(T=$ $300 K)$, respectively. The solid lines represent a least-square fits using the Pässler models [Eq.(2)] while the dash-dotted curves represent least-square fits using the Pässler model minus the thermal expansion contribution of the lattice.

is raised from 0 to $300 \mathrm{~K}$ [54]. On the other hand, Hennel [55] obtained the dilation and vibrational contribution to the temperature shifts of the band-gap energy by considering the temperature dependence of the slop (dE/dT vs. T) of the component parts and the original curve. This procedure resulted in a thermal expansion contribution to the GaAs of $33 \%$. More recently, Mannogian [56] criticized 
the procedure adopted by Hennel in obtaining the thermal dilation contribution to GaAs. A larger value, $44 \%$, was obtained for the thermal dilation contribution to the GaAs at $\mathrm{T}=300 \mathrm{~K}$ [9]. The calculations used to obtain the value of $44 \%$ were based on the data listed in Table I in Biernacki's et al. article [34], which used $\alpha_{t h}$ as constant at T $=300 \mathrm{~K}$ to estimate $\Delta E_{t h}(T)$, and the experimental values $\mathrm{dE} / \mathrm{dT}$, at room temperature; in a similar procedure to the one adopted by Hennel. High values of the thermal dilation contribution were also obtained for other semiconductor materials with the constant value of the linear thermal expansion coefficient, $\alpha_{t h}(T=300 K)$. For example, Hang et al. [8] have estimated in $45 \%$ the thermal dilation contribution to the shift of the band-gap energy in InGaAs at $\mathrm{T}=873 \mathrm{~K}$. Using the adjustment parameters obtained from fittings in Ref. [8], we verified that the $45 \%$ value can be obtained even at $300 \mathrm{~K}$, remaining practically constant at higher temperatures. Using the expression (3) and the thermal expansion coefficient, $\alpha_{t h}(T)$, in all the temperature interval $(0-300$ $\mathrm{K})$, we obtained an intermediate value $(21 \%)$ for the contribution of the TE to the band-gap energy of GaAs at $\mathrm{T}=$ $300 \mathrm{~K}$. This result shows the importance of considering the $\alpha_{t h}(T)$ and the experimental value $E_{g}(T)$ of (instead of the slope $\mathrm{dE} / \mathrm{dT}$ ) in all the temperature range when calculating the energy shift associated with the TE, $\Delta E_{t h}(T)$.

As the inset of Fig. 4 shows, the fit curve, after subtracting the thermal expansion contribution with $\alpha_{t h}(T)$, is below the fit curve of the band-gap energy, $E_{g}(T)$, in the range from $23 \mathrm{~K}$ to $95 \mathrm{~K}$. This behavior originates from the NTE and it shows that the NTE induces the gap to vary at higher energies (blueshift) in the temperature range where the NTE takes place. This effect can also be observed in Fig. 3. The difference between the fittings, with or without the thermal expansion contribution, reaches the value of $\sim 1 \mathrm{meV}$ at $\mathrm{T}=$ $65 \mathrm{~K}$. Although this difference is small, it should be taken into account, once other physical phenomena such as the excitons localization by potential fluctuation in semiconductor alloys are commonly used to explain the blueshifts of this magnitude in the variation of the excitonic transitions with temperature [51,57-59]. Although the NTE induces a small blueshift of energy gap in the temperature range from $23 \mathrm{~K}$ to $95 \mathrm{~K}$, the optical transition energy in the GaAs displays a small redshift in this same temperature range. This can be explained by the competition between the NTE and the electron-phonon interaction. Thus, for the GaAs in the temperature range from $23 \mathrm{~K}$ to $95 \mathrm{~K}$, the electron-phonon interaction is more intense than the NTE (above about $60-70 \mathrm{~K}$ the LO phonon contribution becomes important and eventually dominates the shift of the band-gap energy), producing the redshift effect of the optical transition energy observed in Fig. 4, represented by the solid lines. At higher temperatures, the thermal expansion as well as the electron-phonon interaction induce a decrease of the band-gap energy. The total effect is a strong redshift of the band-gap energy (the linear decreasing of the band-gap energy). Some semiconductor materials of the group II-VI, as the CdTe, HgTe and $\mathrm{HgCdTe}$ have a NTE nine times larger than that observed in the GaAs [24]. Therefore, we should expect, in these materials, a much larger blueshift of energy induced by the NTE than that found for the GaAs.

Despite the large number of optical measurements carried out on the AlGaAs, the temperature dependence of the thermal expansion coefficients of this material in the $2-300$ $\mathrm{K}$ temperature range is still unknown. As the thermal expansion coefficient of the AlAs binary also is not known in the range from 2 to $300 \mathrm{~K}$, it is not possible to obtain $\alpha_{t h}(T)$ for the $\mathrm{Al}_{x} \mathrm{Ga}_{1-x} \mathrm{As}$ ternary alloy from a direct linear interpolation between the $\alpha_{t h}(T)$ values of the GaAs and AlAs binary materials. However, from the available data in the literature, it is possible to obtain a reasonable estimate of the behavior of the linear thermal expansion coefficient of the $\mathrm{Al}_{x} \mathrm{Ga}_{1-x}$ As alloy as a function of temperature. This estimate is based on some experimental observations such as: a) GaAs and several other materials of similar crystalline structure such as $\mathrm{Si}, \mathrm{GaSb}$, AlSb, InP, etc. present NTE at low temperatures $[15,18,21]$. b) Ternary materials ( $\mathrm{HgCdTe}$ and $\mathrm{HgZnTe}$ ), developed from binaries (HgTe, CdTe and ZnTe) which present NTE, also present the same behavior at low temperatures [24]. Moreover, the value of the temperature in which the minimum of NTE is observed in the ternary material is practically the same as that observed in the binary materials [24]. c) Some experimental procedures have been shown that the temperature dependence of the lattice constant for the GaAs $[17,60]$ is characterized by a minimum value at about $60 \mathrm{~K}$. This behavior has also been observed for the $\mathrm{Al}_{0.32} \mathrm{Ga}_{0.68} \mathrm{As}$ alloy [17]. However, for this alloy, the minimum value is smaller than that found for the GaAs. d) Multiple quantum wells of $\mathrm{AlGaAs} / \mathrm{GaAs}$ present NTE at low temperatures [60]. It can be observed from the work of Clec'h et al. [60] that the temperature at which the lattice parameter has its minimum value, for the multiple quantum wells of $\mathrm{AlGaAs} / \mathrm{GaAs}$, is practically the same for different aluminum concentrations. This way, the behavior of the $\mathrm{Al}_{x} \mathrm{Ga}_{1-x} \mathrm{As}$ thermal expansion coefficient with the temperature can be estimated from the thermal expansion coefficient from the GaAs in the all temperature range $(2-300 \mathrm{~K})$, from the linear interpolation between the thermal expansion coefficient values of the GaAs [15] and of the AlAs [61] at $\mathrm{T}=300 \mathrm{~K}$ and from the experimental observations mentioned above. Grimmeiss and Monemar have developed a similar procedure to obtain an estimate of the thermal expansion coefficient to the AlAs and AlP binary materials [62].

Figure 5 shows $\alpha_{t h}(T)$ the curve adjusted to fit the GaAs experimental data points and the curve estimated for the $\mathrm{Al}_{x} \mathrm{Ga}_{1-x}$ As alloy at $x=0.17,0.30$ and 0.40 . Experimental data for AlAs from Ref. 61 are also represented at $\mathrm{T}=300 \mathrm{~K}$. In the $\mathrm{Al}_{x} \mathrm{Ga}_{1-x}$ As alloy system the bulk modulus and the pressure coefficient are dependent on the Al concentration. In this work, we used the experimental dependence $B(x)=\left(75.5+3.23 x+7.93 x^{2}-8.66 x^{3}\right)$ $G P a$ to the bulk modulus [63] and by means of linear interpolation between the GaAs and the AlAs pressure coefficients [2], the used pressure coefficient of the alloy was: $(d E / d P)^{\Gamma}=(0.115-0.13 x) e V / G P a$. Similar to the GaAs material shown in Fig. 3B, the results of the band-gap shift for the $\mathrm{Al}_{x} \mathrm{Ga}_{1-x}$ As due to the TE in the two cases, $\Delta E_{t h}(T)$ with $\alpha_{t h}(T)$ and $\Delta E_{t h}(T)$ with $\alpha_{t h}(T=300 K)$, 


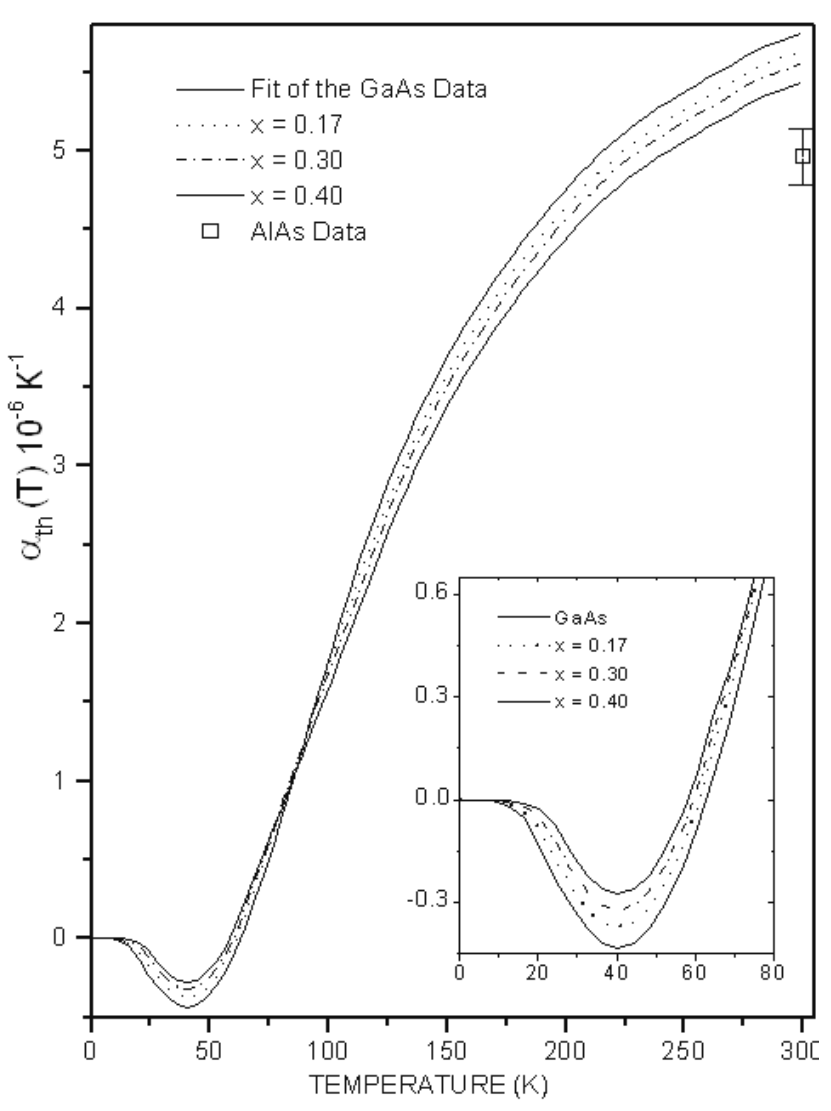

Figure 5. The temperature dependence of linear thermal expansion coefficient for GaAs (thick solid curve) and for AlGaAs alloy at $x=$ 0.17 (doted curve), $x=0.30$ (dash-dotted curve) and $x=0.40$ (fine solid curve). The experimental data for the AlAs, obtained from Ref. 61, are also represented at $T=300 K$. The inset shows the curves obtained in the low temperature interval $(0 K<T<80 K)$.

show a large difference. This difference for the ternary AlGaAs alloy, however, is slightly lower than the difference for the binary GaAs material due to the behavior of $\alpha_{t h}(T)$ with $x$. Figure 6 summarizes the experimental dependence of excitonic transitions with temperature for $\mathrm{Al}_{x} \mathrm{Ga}_{1-x}$ As alloy at $x=0.0,0.17,0.30$ and 0.40 . The curves with close symbols represent the total change of excitonic transitions with temperature. Results for the pure electron-phonon curve are represented by the curves with open symbols. The contribution of the TE mechanism at $\mathrm{T}=300 \mathrm{~K}$, using $\alpha_{t h}(T)$ in the expression (3), corresponds to $24 \%$ at $x=0.17,22 \%$ at $x=0.30$ and $22.3 \%$ at $x=0.40$ of the total variation of the excitonic recombination energy. Thus the variation of the aluminum concentration in the ternary alloy appears not to alter significantly the contribution of the thermal dilatation to the total excitonic transition energy variation with temperature, at least in the range of the aluminum concentration studied. However, according to the model presented for $\alpha_{t h}(x, T)$, the contribution of NTE decreases with increasing aluminum concentration in the $\mathrm{Al}_{x} \mathrm{Ga}_{1-x}$ As alloy.

$\mathrm{Si}$ and Ge electron-phonon spectral functions calculations have shown that the energy shift due to electronphonon interaction includes contributions from both acoustical and optical phonons [35]. Then $\Theta_{B}^{\prime}$ in expression (4) and $\Theta$ in expression (5) should correspond to the average frequency

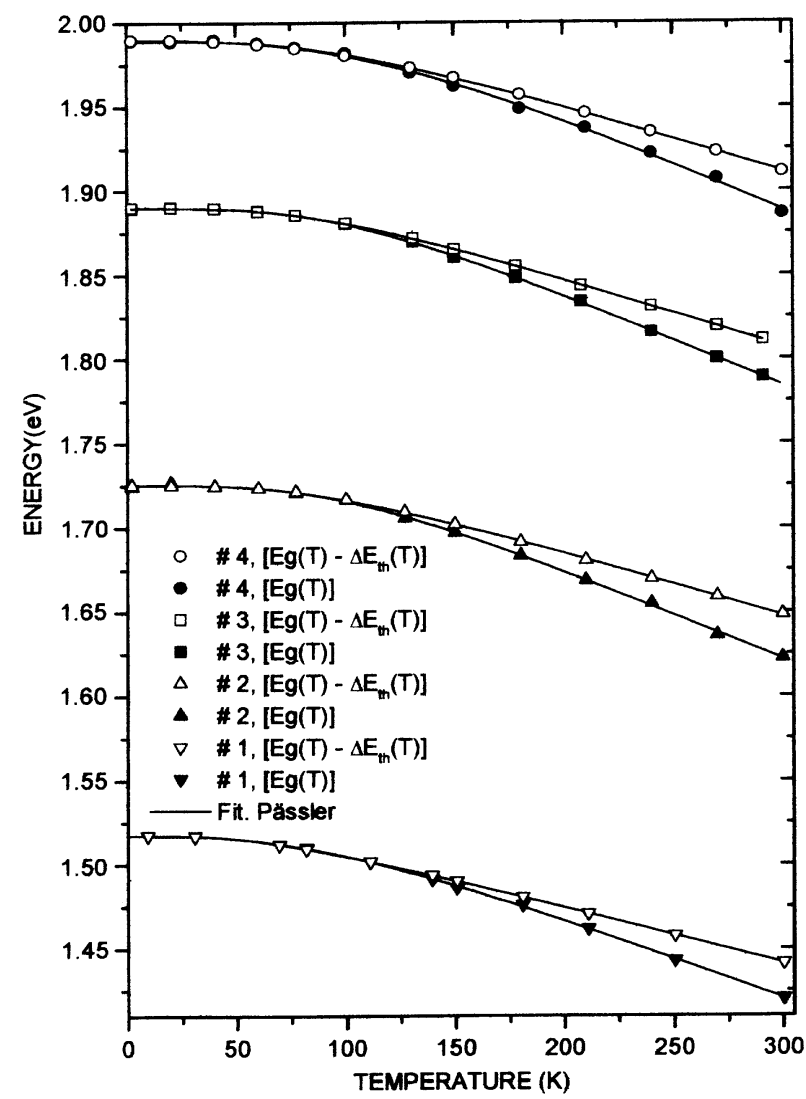

Figure 6. Excitonic transitions in $\mathrm{Al}_{x} \mathrm{Ga}_{1-x}$ As samples with varied aluminum composition are represented as temperature functions. Solid symbols represent experimental data points for the total excitonic transitions variations. Open symbols represent excitonic transitions minus the energy shifts $\Delta E_{t h}(T)$ associated to thermal expansion. The solid lines represent least-aquare fits using the Pässler model [Eq.(2)] or [Eq.(5)].

of the phonon spectra in the temperature scale. However, the electron-longitudinal acoustical phonon interactions give the dominant contribution to the GaAs band-gap energy shift $[9,27,51]$. In the $\mathrm{Al}_{x} \mathrm{Ga}_{1-x} \mathrm{As}$, the energy values associated to $\Theta_{B}$ and $\Theta$ increase due to the variation of the aluminum concentration [11,29]. A recent study showed that the increase of the parameters $\Theta_{B}$ and $\Theta$ due to an increase in the aluminum concentration in the $\mathrm{Al}_{x} \mathrm{Ga}_{1-x}$ As alloy seems to be related only to the optical branches of the phonon spectra [29] (It was shown that the frequency of the longitudinal acoustical (LA) phonon is not dependent on the aluminum concentration in the AlGaAs alloy [64]). However, in the analysis of the parameters $\Theta_{B}$ and $\Theta$, the thermal expansion mechanism and the electron-phonon interaction are not explicitly separated. Table II shows the values for the Viña and Pässler optimized parameters $\left(E_{B}^{,}, a_{B}^{\prime}, \Theta_{B}^{\prime} ; E_{g}^{\prime}(T=\right.$ $\left.0), \alpha^{\prime}, \Theta^{\prime}\right)$ in the GaAs and AlGaAs taking into account the effects of electron-phonon contribution only (i.e. after subtracting the thermal expansion contribution). After the subtraction of the thermal expansion contribution, both the optimized parameters $\left(\Theta_{B}^{\prime}, a_{B}^{\prime}\right.$ and $\left.\Theta^{\prime}, \alpha^{\prime}\right)$ and the effective phonon energy $\left(k_{B} \Theta_{B}^{\prime}\right.$ and $\left.k_{B} \Theta^{\prime}\right)$ also increase with 
increasing of the aluminum concentration. Thus, the longitudinal optical (LO) phonons seem actually to be the main responsible for the increase either of the parameters $\left(\Theta_{B}\right.$ and $\left.\Theta^{\prime}\right)$ or $\left(\Theta_{B}\right.$ and $\left.\Theta\right)$. The optimized parameter related to the strength of the electron-phonon interaction $\left(a_{B}^{\prime}\right)$ also increases with $x$ indicating that the electron-LO phonon interaction, in the $\mathrm{Al}_{x} \mathrm{Ga}_{1-x} \mathrm{As}$ alloy, increases relatively to the electron-LA phonon interaction. This result is in agreement with the results of Kash et al.[65] who, using time-domain pump-probe Raman technique to measure directly the relative strengths of the Fröhlich interaction for "AlAs-like" and "GaAs-like" LO phonons in the two-mode $\mathrm{Al}_{x} \mathrm{Ga}_{1-x} \mathrm{As}$ system, have shown that the relative strength of the electronLO phonon interactions increases with $x$ composition.

\section{Conclusions}

In conclusion, the excitonic transition energy dependence on temperature at different aluminum concentrations in the AlGaAs system was analyzed. The results presented in this work showed that the NTE in the $\mathrm{Al}_{x} \mathrm{Ga}_{1-x}$ As alloy, in the low temperature interval, induces a small blueshift in the optical transition energy. In the temperature range from $\sim 23$ to $\sim 95 \mathrm{~K}$ there is a competition between NTE effect and the electron-phonon interaction. Additionally, we have shown that is necessary to take into account the thermal expansion coefficient in all the temperature range $(0-300 \mathrm{~K})$ to the present analysis of the temperature dependence of the bandgap energy for the GaAs semiconductor materials in the 2 $300 \mathrm{~K}$ temperature range, and that the variation of the aluminum concentration, in the ternary alloy $\mathrm{Al}_{x} \mathrm{Ga}_{1-x} \mathrm{As}$, appears not to alter significantly the contribution of the TE to the total variation of the excitonic transitions energy with temperature.

Additionally, we have shown indirectly that the strength of the electron-LO phonon interaction, relatively to the electron-LA phonon interaction, increases with increasing $A l$ concentration in the AlGaAs alloy.

\section{Acknowledgments}

This work was partially supported by the Brazilian agencies: CAPES, CNPq, and FBB.

The authors would like to thank Professor Dr. Irineu Mazzaro, Departamento de Física, Universidade Federal do Paraná, Brazil for X-ray diffraction measurements, and to Professor Dr. E. Arraes Meneses, Instituto de Física Gleb Wataghin - IFGW, UNICAMP, Brazil for having allowed us to use the facilities of the Laboratory of Optical Properties, where the photoluminescence measurements were carried out.

\section{References}

[1] H. Morkoç and H. Unlu, Semiconductors and Semimetals, (Edited by R. Dingle), (Academic Press, San Diego, 1987), Vol. 24, Chapter 2.
[2] S. Adachi, J. Appl. Phys. 58, R1 (1985); I. Vurgaftman, J. R. Meyer, and L. R. Ram-Mohan, J. Appl. Phys. 89, 5815 (2001), and references therein.

[3] P. B. Allen and V. Heine, J. Phys. C: Solid State 9, 2305 (1976).

[4] S. Zollner, S. Gopalan, and M. Cardona, Solid State Commun. 77, 485 (1991), and references therein.

[5] P. B. Allen and M. Cardona, Phys. Rev. B 23, 1495 (1981).

[6] A. Manoogian and J. C. Wolley, Can. J. Phys. 62, 285 (1984).

[7] J. P. Walter, R. R. L. Zucca, M. L. Cohen, and Y. R . Shen, Phys. Rev. Lett. 24, 102 (1970).

[8] Z. Hang, D. Yan, F. H. Pollak, G. D. Pettit, and J. M. Woodall, Phys. Rev. B 44, 10546 (1991).

[9] R. Pässler, Phys. Status. Solidi. B 200, 155 (1997).

[10] M. El Allali, C. B. Sorensen, E. Veje, and P. TidemandPetersson, Phys. Rev. B 48, 4398 (1993).

[11] S. Logothetidis, M. Cardona, and M. Garriga, Phys. Rev. B 43, 11950 (1991).

[12] E. Grilli, M. Guzzi, R. Zanboni, and L. Pavesi, Phys. Rev. B 45, 1638 (1992).

[13] H. Shen, S. H. Pan, Z. Hang, J. Leng, F. H. Pollak, J. M. Woodall, and R. N. Sacks Appl. Phys. Lett. 53, 1080 (1988).

[14] C. H. Xu, C. Z. Wang, C. T. Chan, and K. M. Ho, Phys. Rev. B 43, 5024 (1991).

[15] T. Soma, J. Satoh, and H. Matsuo, Solid State Commun. 42, 889 (1982).

[16] S. Biernacki and M. Scheffler, Phys. Rev. Letters, 63, 290 (1989).

[17] M. Leszczynski, V. B. Pluzhnikov, A. Czopnik, J. BakMisiuk, T. Slupinski, J. Appl. Phys. 82, 4678 (1997).

[18] K. G. Lion, G. L. Salinger, C. A. Swenson, G. K. White, J. Appl. Phys. 48, 865 (1977).

[19] G. Dolling and R. A. Cowley, Proc. Phys. Soc. London 88, 463 (1966)

[20] H. Ibach, Phys. Status Solidi 31, 625 (1969).

[21] S. I. Novikova, N. Kh. Abrikhosov; Sov. Phys. Solid State (English Transla.) 5, 1558 (1963); Fiz. Tverd. Tela 5, 2138 (1963).

[22] P. W. Sparks, C. A. Swenson, Phys. Rev. 163, 779 (1967).

[23] D. F. Gibbons, Phys. Rev. 112, 136 (1958).

[24] D. Bagot, R. Granger, and S. Rolland, Phys. Stat. Sol. (b) 177, 295 (1993).

[25] Viña, S. Logothetidis, and M. Cardona, Phys. Rev. B 30, 1979 (1984).

[26] R. Pässler, G. Oelgart, J. Appl. Phys. 82, 2611 (1997).

[27] P. Lautenschlager, M. Garriga, S. Logothetidis, and M. Cardona, Phys. Rev. B 35, 9174 (1987).

[28] [28] I. C. Bassignana, D. A. Macquistan, R. W. Streater, G. C. Hillier, R. Packwood, and V. Moore, J. Cryst. Growth. 172, 25 (1997).

[29] S. A. Lourenço, I. F. L. Dias, J. L. Duarte, E. Laureto, E. A. Menese, J. R. Leite, and I. Mazzaro, J. Appl. Phys. 89, 6159 (2001).

[30] C. D. Thurmond, J. Electrochem, Soc. 122, 1133 (1975). 
[31] R. Pässler, Solid State Electronics 39, 1311 (1996).

[32] R. Pässler, J. Appl. Phys. 83, 3356 (1998).

[33] L. Malikova, W. Krystek, F. H. Pollak, N. Dai, A. Cavus, and M. C. Tamargo, Phys. Rev. B 54, 1819 (1996).

[34] S. Biernacki, U. Scherz, and B. K. Meyer, Phys. Rev. B 49, 4501 (1994).

[35] P. Lautenschlager, P. B. Allen, and M. Cardona, Phys. Rev. B 31, 2163 (1985).

[36] V. Swaminathan, J. L. Zilko, W. T. Tsang, and W. R. Wagner, J. Appl. Phys. 53, 5163 (1982).

[37] Y. Chen, G. P. Kothiyal, J. Singh, and P. K. Bhattacharya, Superlatt. Microstruct 3, 657 (1987).

[38] H. Heilman and G. Oelgart, Semicond. Sci. Technol. 5, 1040 (1990).

[39] D. E. Aspnes, Surf. Sci. 37, 418 (1973).

[40] F. Cerdeira, Braz. J. Phys. 23, 3 (1993).

[41] F. G. Sánchez-Almazan, H. Navarro-Contreras, G. Ram'rezFlores, and M. A. Vidal, J. Appl. Phys. 79, 7713 (1996).

[42] R. A. Kuzmenko and É. P. Domashevskaya, Semiconductors 36, 278 (2002).

[43] O. J. Glembocki, N. Bottka, and J. E. Furneaux, J. Appl. Phys. 57, 432 (1985).

[44] S. K. Brierley and D. S. Lehr, J. Appl. Phys. 67, 3878 (1990), and references therein.

[45] A. V. Ganzha, R. V. Kuzmenko, W. Kircher, J. Screiber, and S. Hildebrandt, Semiconductors 32, 245 (1998), and references therein.

[46] R. Wang and D. Jiang, J. Appl. Phys. 72, 3826 (1992).

[47] R. Kuzmenko, A. Ganzha, J. Schreiber, and S. Hildebrandt, Phys. Solid State 39, 1900 (1997).

[48] E. F. Schubert and W. T. Tsang, Phys. Rev. B 34, 2991 (1986).

[49] W. Shan, W. Walukiewicz, E. E. Haller, B. D. Little, J. J. Sang, M. D. McCluskey, N. M. Johnson, Z. C. Feng, M. Schurman, and R. A. Stall, J. Appl. Phys. 84, 4452 (1998).
[50] F. Schubert, E. O. Göbel, Y. Horikoshi, K. Ploog, and H. J. Queisser, Phys. Rev. B 30, 813 (1984).

[51] B. Q. Sun, D. S. Jiang, Z. Pan, L. H. Li, and R. H. Wu, Appl. Phys. Leet. 77, 4148 (2000).

[52] C. Lárez and C. Rincón, J. Phys. Chem. Solids, 58, 1111 (1997).

[53] G. Fonthal, L. Tirado-Méjía, J. I. Marin-Hurtado, H. ArizaCalderón, J. G. Mendoza-Alvarez, J. Phys and Chemistry of Solids 61, 579 (2000).

[54] S. Gopalan, P. Lautenschlager, and M. Cardona, Phys. Rev. B 35, 5577 (1987).

[55] A. M. Hennel, Phys. Stat. Sol. A, 8, K111 (1971).

[56] A. Manoogian Can. J. Phys. 60, 1490 (1982).

[57] D. Ouadjaout and Y. Marfaing, Phys. Rev. B 46, 7908 (1992).

[58] H. P. D. Schenk, M. Lerox, and P. de Mierry, J. Appl. Phys. 88, 1525 (2000).

[59] S. A. Lourenço, I. F. L. Dias, L. C. Poças, J. L. Duarte, J. B. B. de Oliveira, and J. C. Harmand, J. Appl. Phys. 93, 4475 (2003).

[60] G. Clec'h, G. Calvarin, P. Auvray, and M. Baudet, J. Appl. Crystallogr. 22, 372 (1989).

[61] I. I. Pashintsev, N. N. Sirota; Dokl. Akad. Nauk SSSR 3, 38 (1959).

[62] H. G. Grimmeiss and B. Monemar, Phys. Stat. Sol. (a) 5, 109 (1971).

[63] S. Gehrsitz, H. Sigg, N. Herres, K. Bachem, K. Köhler, and F. K. Reinhart, Phys. Rev. B 60, 11601 (1999).

[64] L. Pavesi, and M. Guzzi, J. Appl. Phys. 75, 4779 (1994), and references therein.

[65] J. A. Kash, S. S. Jha, and J. C. Tsang, Phys. Rev. Lett. 58, 1869 (1987). 